\title{
Stability Analysis of Tubular Steel Shores
}

Fábio André Frutuoso Lopes, Fernando Artur Nogueira Silva, Romilde Almeida de Oliveira and Romildo Alves Berenguer

Department of Civil Engineering, Catholic University of Pernambuco, Recife 51110-200, Brazil

\begin{abstract}
The design of tubular steel scaffold-type shoring is usually performed by calculating the load capacity of the elements, taking into account their axial strength, mainly. Geometric stiffness effects and changes in the stiffness of connections are seldom considered. This paper assesses the stability of tubular steel shores using experimental and numerical approaches that take into account geometric nonlinearities as well as the features of the elements used to make the link between the steel tubes (pressed double coupler-right angle). The increase in overall stiffness generated by diagonal bars used in the analyzed models was examined. The results obtained show the importance of using P-delta analyses in this kind of structure in order to evaluate structure's overall stability even when compressive stresses are within acceptable ranges of code limits.
\end{abstract}

Key words: Tubular steel shores, global stability, semi-rigid connections, P-delta analyses.

\section{Introduction}

Steel shores must be designed and built to support the weight of structures during construction and should have adequate stiffness to ensure the integrity of the structural elements that they temporarily support. Under their self-weight, the weight of the supported structure and building live loads, steel shores must not display large deflections that can affect structure functionality and should not generate stress resultants, which have not been considered in the design.

The design of tubular steel scaffold-type shoring is usually performed taking into account the individual elements' axial load capacity only by adopting pin support ends as boundary conditions. If the global stability of the structure is ensured, this design approach is realistic enough, but depending on shore stiffness, the ultimate limit state is not governed by the buckling of isolated compressed elements and the structure's global buckling becomes more important. In this situation, the elements' unsupported length can not be accurately estimated because connections of the coupler are of varying degrees of fixity and some

Corresponding author: Romildo Alves Berenguer, professor, research fields: concrete, corrosion materials and structural concrete. bending at the joints can occur. This way, the effective lengths of elements can not be calculated considering exclusively the distance between horizontal coupler elements and the stiffness generated by element geometries and their connection must be taken into account.

This paper discusses the importance of considering second-order effects in the design of tubular steel scaffold-type shoring. The assembly of scaffold frames in tower structures with different overall stiffness was modeled in order to study and evaluate its flexural behavior using real in-situ features of the connections. In the design of steel frame structures, it is usual to consider the connections as flexible (perfect hinges) or as rigid ones (fully rigid), although in practice joints are neither ideally hinged nor rigid. In fact, all the joints exhibit some relative rotation between the members being joined and this is due to the deformation of elements in the joint. Thus, it would be more appropriate to model these connections as semi-rigid elements (partially restrained), because this hypothesis leads to potential economies that can be achieved from simpler connection details, adjusting their stiffnesses to optimize the distribution of moments in the connected 
members. For design purposes, any joint that develops more than $90 \%$ of the ideal rigid joint moment can be classified as rigid and those that develop less than $20 \%$ of the ideal rigid joint moment should be considered as hinged. Joints that develop bending moments between these two values are considered semi-rigid [1,2]. Fig. 1 depicts this concept showing in a schematic way curves for each type of connection and a straight line that represents the relationship between end-moments and rotation for a simply supported and uniformly loaded beam.

To calculate the relationship between the end-bending moments and rotations, tests were performed to evaluate the efficiency of orthogonal coupler. Table 1 shows the properties of the steel tubes used in tests and numerical simulations.

\section{Load Capacity According to Brazilian Code}

The design strength [3] for a compressed structural member subject to flexural and local buckling can be

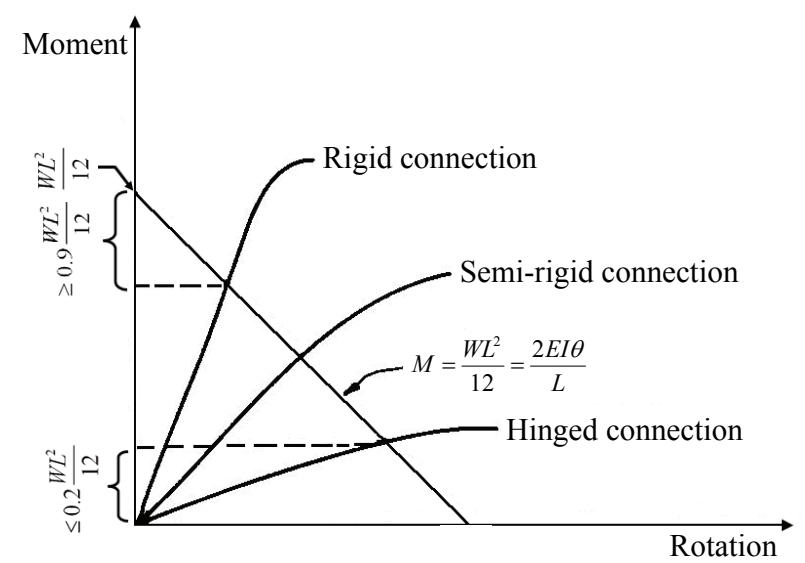

Fig. 1 Moment versus joint rotation [1].

Table 1 Properties of steel tubes used.

\begin{tabular}{ll}
\hline Properties of the steel tubes & \\
\hline Outside diameter $(\mathrm{mm})$ & 48.30 \\
Thickness $(\mathrm{mm})$ & 3.05 \\
Cross section area $\left(\mathrm{mm}^{2}\right)$ & 433 \\
Yield stress $\left(f_{y}\right)(\mathrm{MPa})$ & 400 \\
Young's modulus $(\mathrm{GPa})$ & 205 \\
Specific weight $\left(\mathrm{kN} / \mathrm{m}^{3}\right)$ & 77 \\
Poisson ratio $(v)$ & 0.30 \\
\hline
\end{tabular}

calculated using Eq. (1) and the slenderness ratio can be obtained using Eq. (2) as follows:

$$
\begin{gathered}
N_{n}(L)=\emptyset_{c} \rho(L) Q A_{g} f_{y} \\
\lambda(L)=\frac{K L}{r}
\end{gathered}
$$

where:

$\varnothing_{c}=$ resistance factor;

$\rho(L)=$ factor related to flexure buckling;

$Q=$ reduction factor due to local buckling;

$A_{g}=$ gross area of member, $\mathrm{cm}^{2}$;

$f_{y}=$ yield stress of steel, $\mathrm{kN} / \mathrm{cm}^{2}$;

$K=$ effective length factor;

$L=$ length of member, $\mathrm{cm}$;

$r=$ radius of gyration, $\mathrm{cm}$.

\section{Numerical Modeling}

To evaluate the influence of the degree of fixity generated by couplers on global stability, models with different boundary conditions were studied (Fig. 2). In the analyses, the joints were modeled as semi-rigid connections and their rotational stiffnesses were calculated from experimental tests described in the next section. Software SAP2000 was used in numerical modeling [4].

Two boundary conditions were considered:

- translation restraints only on base nodes;

- translation restraints in all nodes on the base and on the top.

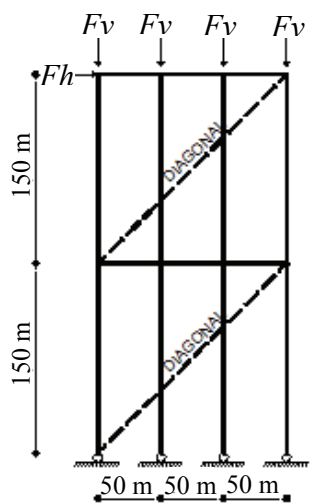

(a)

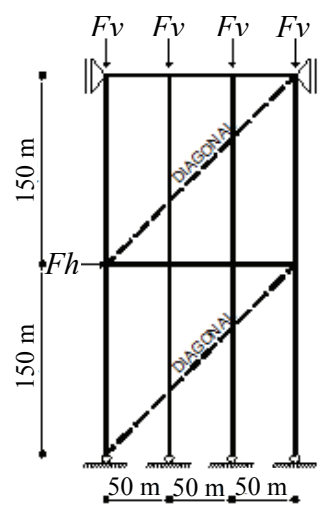

(b)
Fig. 2 Boundary conditions of the Models (a) and (b): (a) translation restraints only on base nodes; (b) translation restraints in all nodes on the base and on the top. 
P-delta analyses were performed using a convergence criterion of $1 \%$ for displacements and force tolerance and the maximum number of iteration was set to 10. To start the iteration process, the structures were subjected to an initial eccentricity and a lateral load equal to $0.1 \%$ of nodal vertical load, according to Ref. [5]. In the structure that has lateral displacement restraints on the base and on the top, this force was applied in a central node; and in the structure whose only displacement restraints are located on its base, this nodal force was applied to a top node.

Applying the boundary conditions described above on Models (a) and (b) from Fig. 3 results in four structures to be analyzed. One that comes from the use of rigid connections on Model (a) and three other structures considering hinged, semi-rigid or rigid connections on Model (b).

The vertical load was calculated adopting a value of $30 \%$ of the load capacity of the tube according to Eq. (1), considering a member buckling length of $1.5 \mathrm{~m}$. This way, the vertical and horizontal load used in numerical analyses were respectively $20.4 \mathrm{kN}$ and $0.02 \mathrm{kN}$. Additionally, the influence of diagonal members on the behavior of the structure with hinged connections as compared with the other structures modeled was studied.

\section{Tests Performed to Calculate Rotational Brace Stiffness}

The degree of fixity of a connection should not be calculated from the moment-rotation relationship only because this degree is not enough to classify the connection as rigid or as hinged. This classification can only be done taking into account the relationship between the coupler stiffness and the flexural stiffness of the elements they connect. This ratio is often called "common stiffness" and can be expressed through Eq. (3):

$$
\frac{k L}{E I}
$$

where:

$k=$ coupler rotational stiffness;

$L=$ length of member connected to coupler;

$E=$ modulus of elasticity;

$I=$ moment of inertia.

Fig. 4 shows details of the tests performed to calculate coupler flexural rotational stiffness. Loads were applied using a hydraulic jack with a capacity of $200 \mathrm{kN}$, provided with a calibrated pressure transducer cell capable of reading forces up to $40 \mathrm{kN}$. Digital deflectometers with a precision of $0.003 \mathrm{~mm}$ were used to measure linear displacements. Five orthogonal couplers made with hardened forged spring steel were tested. When these couplers are subjected to the tubes' flexure, they offer a torsion reaction. The distance between the axes of the tubes engaged was $65 \mathrm{~mm}$. Properties of the steel tubes used in tests and numerical simulations are those in Table 1. Vertical and horizontal steel bars used in experimental models have the same properties.

In order to minimize displacements due to axial deformations during the loading process, steel tubes with solid cross sections were used in the tests. Fig. 3 shows moment $x$ rotation curves for each one of tested couplers from which was derived a function $M(x)$ to calculate the degree of torsion fixity of the couplers. The secant stiffness used in numerical modeling was defined in a way that the number considered was representative of all tested specimens. In this way, it was assigned a $0.04 \mathrm{rad}$ of relative rotation to all

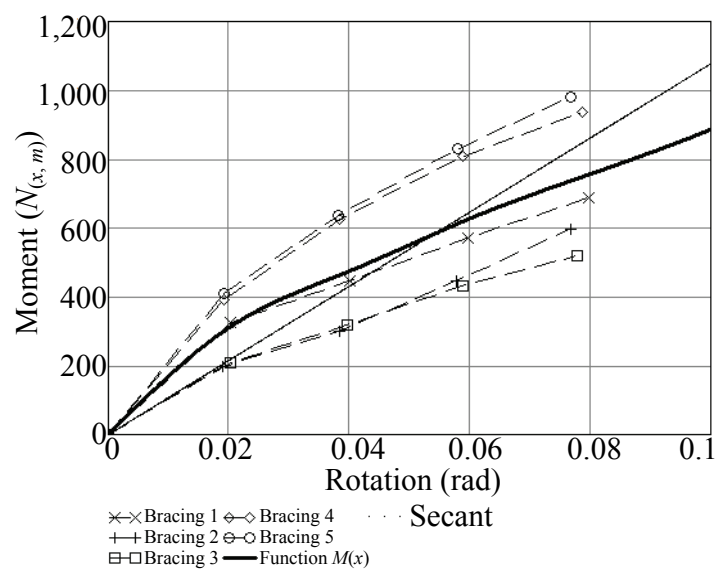

Fig. 3 Moment $x$ rotation curves. 


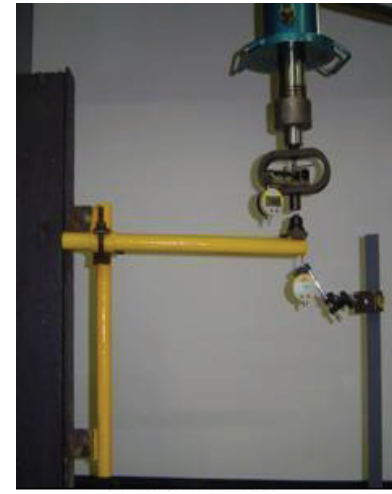

(a)

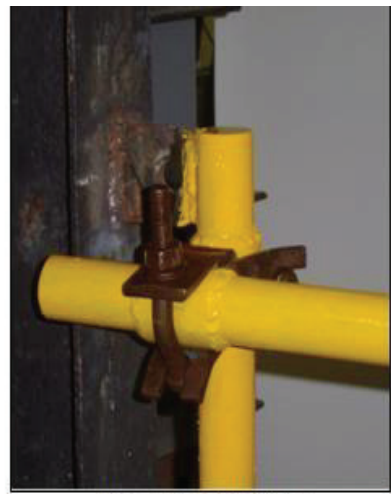

(b)

Fig. 4 Test details: (a) test performed; (b) pressed coupler detail.

frames of the model which corresponds to a torsional stiffness of $12.1 \mathrm{kNm} / \mathrm{rad}$. The definition of secant stiffness used was an approximation to represent a safe behavior for all the couplers tested, since the use of initial stiffness would generate underestimated displacements.

Fig. 5 shows lateral displacements from P-delta analysis on the nodes (where horizontal forces were applied) versus the number of panels. One can verify that for the two studied models, the structures with flexible nodes with added diagonal bars exhibited larger lateral stiffness than those structures modeled considering rigid connections only.

Fig. 3 presents the second-order horizontal displacement curves derived from the analysis performed in Model (b), taking into account the hinged connection approach with a rotational constant spring value equal to $12.1 \mathrm{kNm} / \mathrm{rad}$. Since the torsional stiffness used in numerical modeling offers little rotational fixity, both models presented equivalent lateral stiffness as can be seen in Fig. 3 .

One important fact that should be observed was the dispersion of moment $x$ rotation curves obtained from the tests with the couplers. This fact possibly occurred due to difficulties in controlling the pressure applied on the screws of the coupled system of orthogonal connections.

Analyzing the results in Fig. 6, one can observe that the connections modeled generated on the structure stiffness similar to those obtained for a system modeled

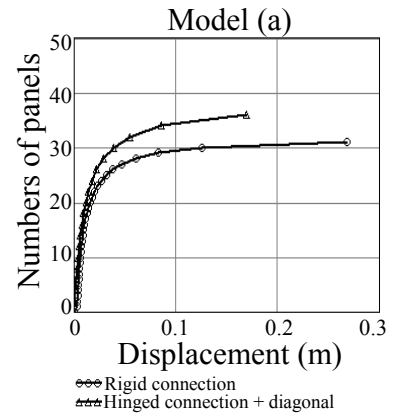

(a)

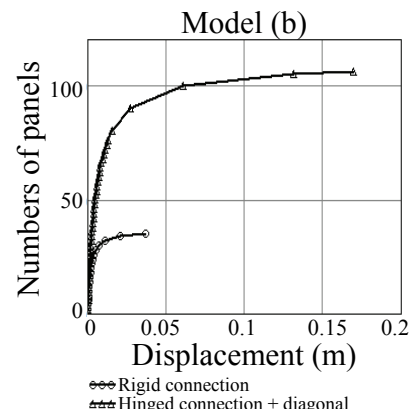

(b)
Fig. 5 Non-linear geometric behavior of Models (a) and (b): (a) test performed; (b) pressed coupler detail.

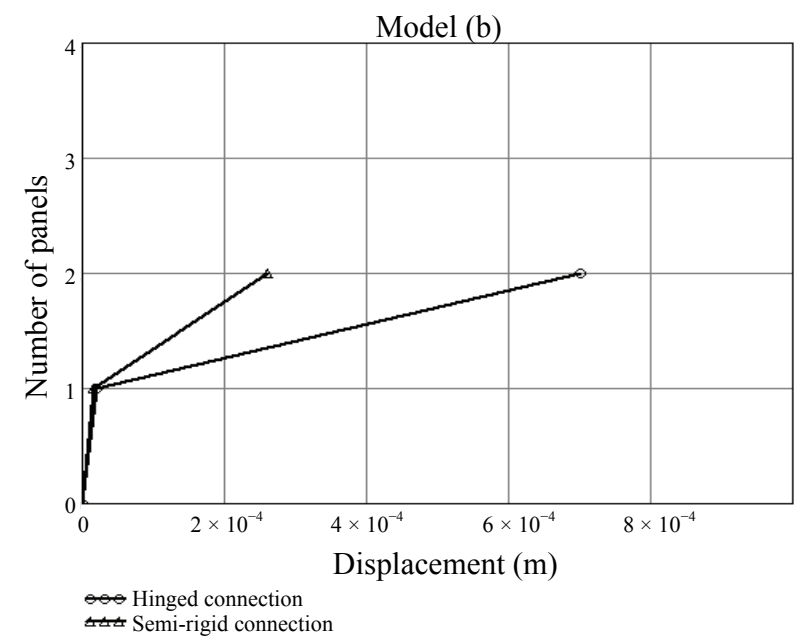

Fig. 6 Behavior of Model (b) using the hinged connections without diagonal bars and with rotational constant spring value of the tested couplers.

with hinged connections on its ends. Thus, second-order analyses applied on Model (b) with hinged connections or using the stiffness torsional constant obtained with the tests performed converge only for two panels.

\section{Conclusions}

Based on the data developed in this investigation, the following conclusions could be drawn:

(1) The torsional stiffness value of couplers obtained from experimental tests generated for the studied models a degree of fixity in their connections similar to those that can be obtained adopting the hinged connections approach. However, it is important to highlight that the couplers used on the tests were not brand new. Conversely, they presented some degree of abrasion from their use and were 
chosen because they represent real conditions usually found in steel shoring works;

(2) Diagonal bars added to structures with hinged connections produced a lateral stiffness larger than that obtained on models with rigid connections without diagonal bars. This increase was larger in Model (b).

The results obtained should be applied with caution, especially those represented in Figs. 5 and 6, and must be seen as an estimation of the lateral stiffness of the analyzed models. It is not possible to extrapolate directly to design situations because the load conditions used are not those usually found in steel shoring structures.

\section{References}

[1] CBCA (Brazilian Centre of Steel Construction). 2004.
Steel Structure Connections. Rio de Janeiro: CBCA. (in Portuguese)

[2] Ferreira, M. A., El Debs, M. K., and Elliot, K. S. 2003. "Theoretical and Experimental Determination of Moment-Rotation Relationship in Beam-Column Connections in Precast Concrete Structures." Presented at 5th Symposium EPUSP (Escola Politécnica da Universidade de São Paulo) on Concrete Structures, São Paulo, Brazil. (in Portuguese)

[3] ABNT (Brazilian Association of Technical Codes). 2008. NBR 8800: Design and Construction of Steel Buildings Structures (Limit State Method). Rio de Janeiro: ABNT. (in Portuguese)

[4] Computers and Structures, Inc. 2014. SAP2000-Integrated Finite Element Analysis and Design of Structures. California: Computers and Structures, Inc.

[5] Lopes, A. P., Santos, G. O., and Souza, A. L. A. C. 2005. "Study about Different Methods of P-delta Analysis." Presented at 47th IBRACON (Instituto Brasileiro do Concreto) Conference, Recife, Brazil. (in Portuguese) 